\title{
Influence des activités humaines sur la répartition des fourmis du genre Messor dans les Pyrénées-Orientales (Hymenoptera : Formicidae : Myrmicinae)
}

\author{
Claude LEBAS ${ }^{1}$ iD
}

LEBAS, C. (2021). Influence des activités humaines sur la répartition des fourmis du genre Messor dans les Pyrénées-Orientales (Sud de la France) (Hymenoptera : Formicidae : Formicinae). Osmia, 9: 65-76. https://doi.org/10.47446/OSMIA9.9

\begin{abstract}
Résumé
En se fondant sur la répartition du genre Messor dans les Pyrénées-Orientales (France), une étude de la distribution et de la structuration géographique du genre est présentée, accompagnée d'une discussion du processus de dispersion au cours des âges. Ces fourmis ont un régime alimentaire strictement composé de graines dont la disponibilité influence leur implantation. Un lien entre le développement historique de l'agriculture humaine, caractérisé par un déplacement concomitant des plantes cultivées et messicoles, et la distribution géographique des fourmis Messor est suggéré dans cette étude.
\end{abstract}

Mots-clefs | Mer méditerranée $\cdot$ Sud de la France $\cdot$ granivorie $\cdot$ phylogéographie $\cdot$ biogéographie $\cdot$ Messor barbarus $\cdot$ Messor bouvieri $\cdot$ Messor capitatus $\cdot$ Messor ibericus

Influence of human activities on the distribution of ants of the genus Messor in Pyrénées-Orientales (Hymenoptera: Formicidae: Myrmicinae)

\begin{abstract}
Based on the distribution of the genus Messor in Pyrénées-Orientales (France), a study of the distribution and geographical structure of the genus is presented, accompanied by a discussion of the process of dispersal over the ages. These ants have a diet strictly composed of seeds, the availability of which influences their establishment. A link between the historical development of human agriculture, characterized by concomitant displacement of cultivated and messicole plants, and the geographic distribution of Messor ants is suggested in this study.
\end{abstract}

Keywords $\mid$ Mediterranean Sea $\cdot$ Southern France $\cdot$ granivory $\cdot$ phylogeography $\cdot$ biogeography $\cdot$ Messor barbarus $\cdot$ Messor bouvieri $\cdot$ Messor capitatus $\cdot$ Messor ibericus

Reçu•Received | 03 April 2021 || Accepté • Accepted| 20 November 2021 || Publié (en ligne) • Published (online)| 24 November 2021 Reviewers | P. BOURLET • L. COLINDRE || http://zoobank.org/9EFB3C44-7F98-4579-ADDF-8C7DCD39C974

\section{INTRODUCTION}

Lors de mes inventaires et sorties d'étude de la myrmécofaune en France et en Europe du Sud, j'ai pu constater la récurrence de la présence des fourmis moissonneuses Messor capitatus sur des lieux liés à l'histoire humaine, comme des chapelles ou des sites antiques, à l'écart de l'urbanisation récente. Cette observation m'a conduit à mener un inventaire plus poussé des fourmis du genre Messor dans la plaine du Roussillon avec cette question: quels intérêts trouveraient des fourmis granivores à établir leur nid sur des sites historiquement anthropisés ?

Pour y répondre, il convient de faire préalablement le point sur les préférences écologiques connues chez les espèces du genre Messor, représenté en France par sept espèces (LEBAS et al., 2016). Pour établir leur répartition, je me suis basé sur une synthèse bibliographique (notamment ARMAN et al., 2010 ; BERNARD, 1985 ; BLATRIX et al., 2016 ; CAGNIANT, 1962, 1973 ; CAGNIANT \& ESPADALER, 1998 ; COLLECTIF, 2021a) et plus encore sur des observations personnelles ou communiquées par des collègues. Ces données avaient déjà constitué la base des cartes illustrant le guide des Fourmis d'Europe occidentale (LEBAS et al., 2016).

Parmi les espèces françaises, il apparaît ainsi que :

- deux espèces ne se trouvent qu'en Corse : Messor wasmanni KRAUSSE, 1910 (figures 1-2) et Messor minor (ANDRÉ, 1881) (figure 3-4). 


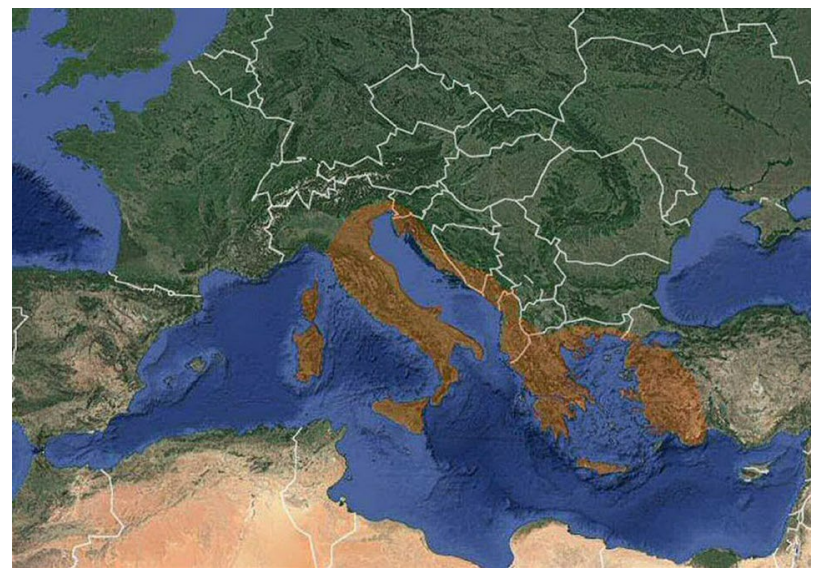

Figure 1. Répartition globale de Messor wasmanni.

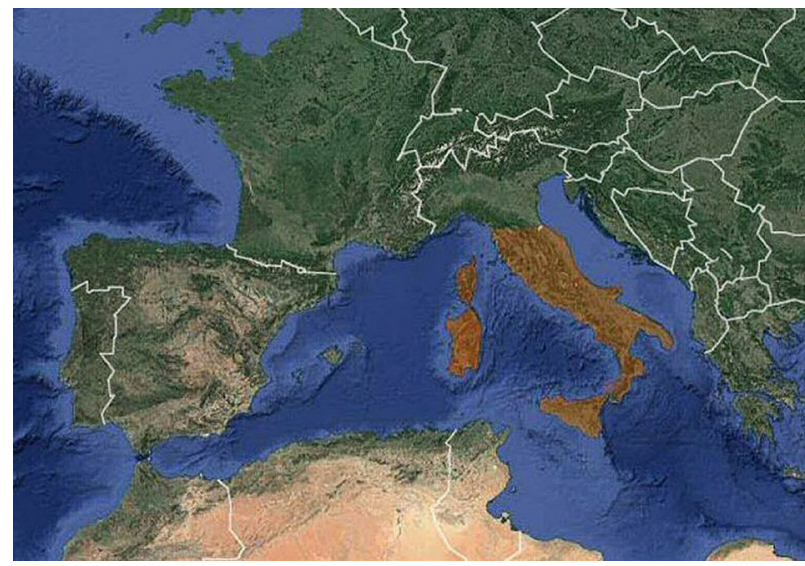

Figure 3. Répartition globale de Messor minor.

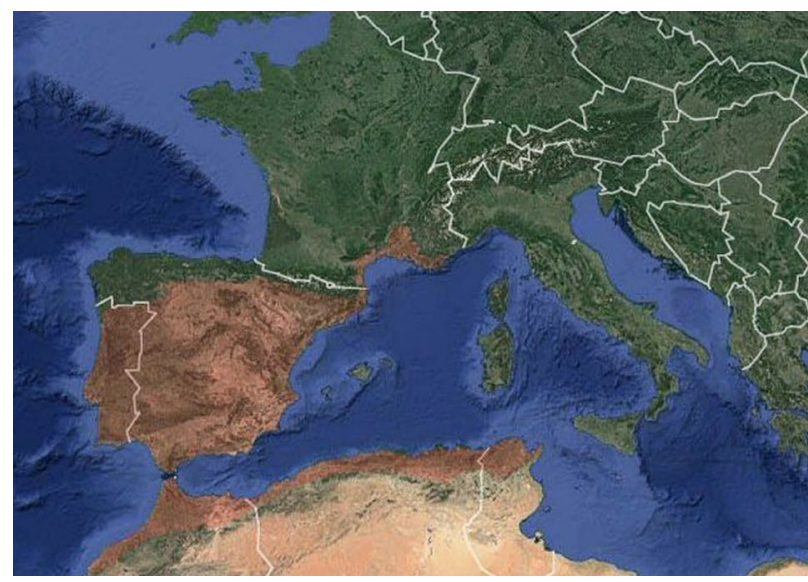

Figure 5. Répartition globale de Messor barbarus.

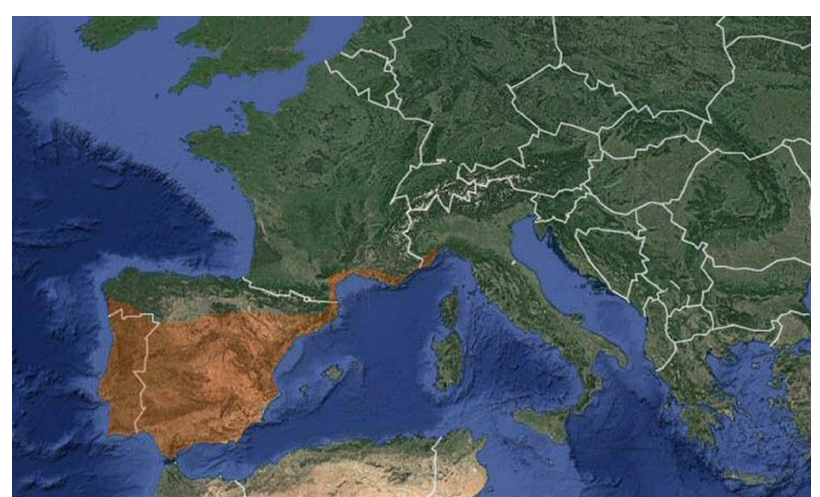

Figure 7. Répartition globale de Messor bouvieri.

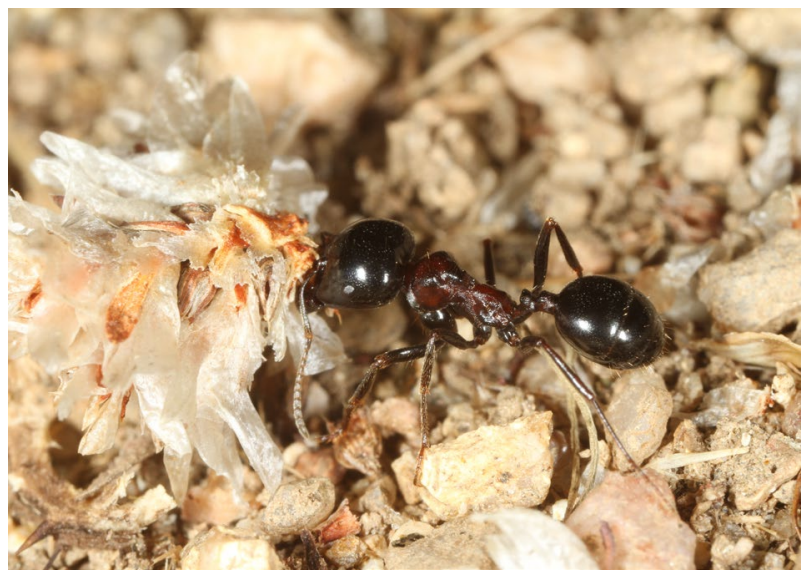

Figure 2. Ouvrière de Messor wasmanni. Cliché C. LEBAS.

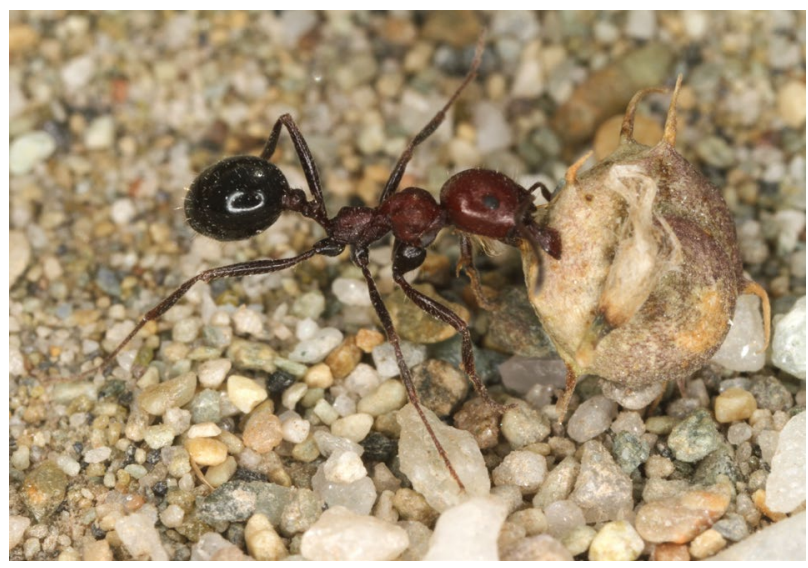

Figure 4. Ouvrière de Messor minor. Cliché C. LEBAS.

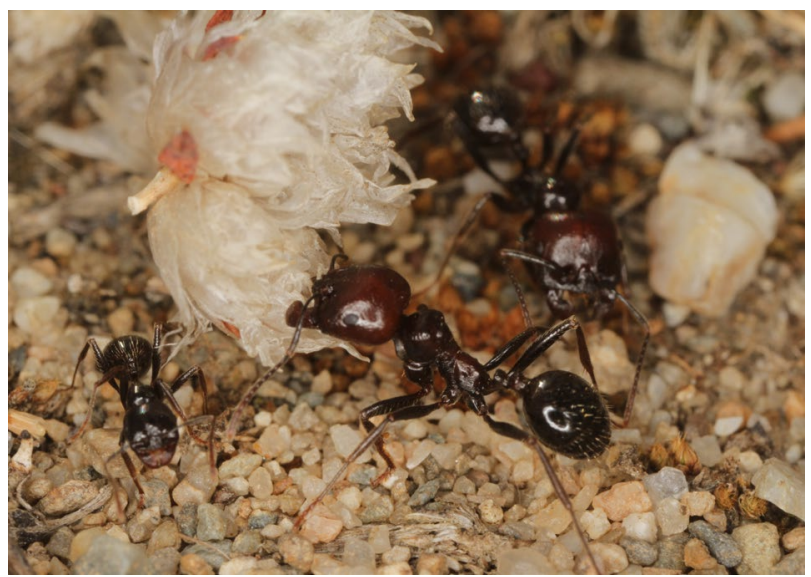

Figure 6. Ouvrière de Messor barbarus. Cliché C. LEBAS.

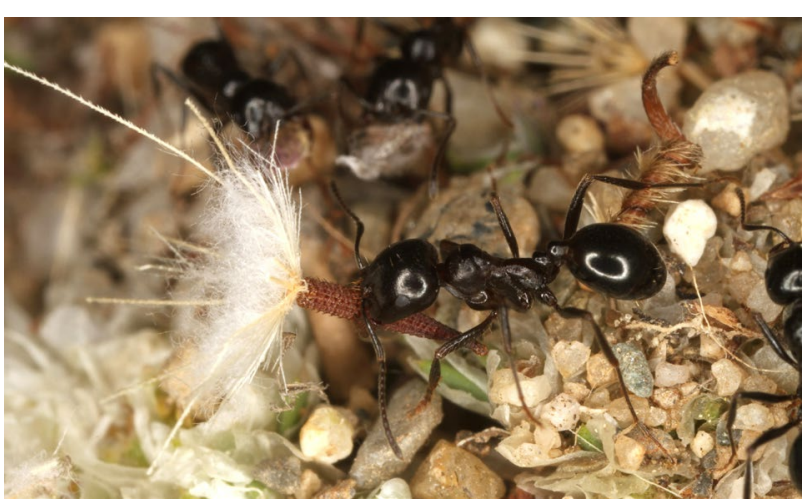

Figure 8. Ouvrière de Messor bouvieri. Cliché C. LEBAS. 


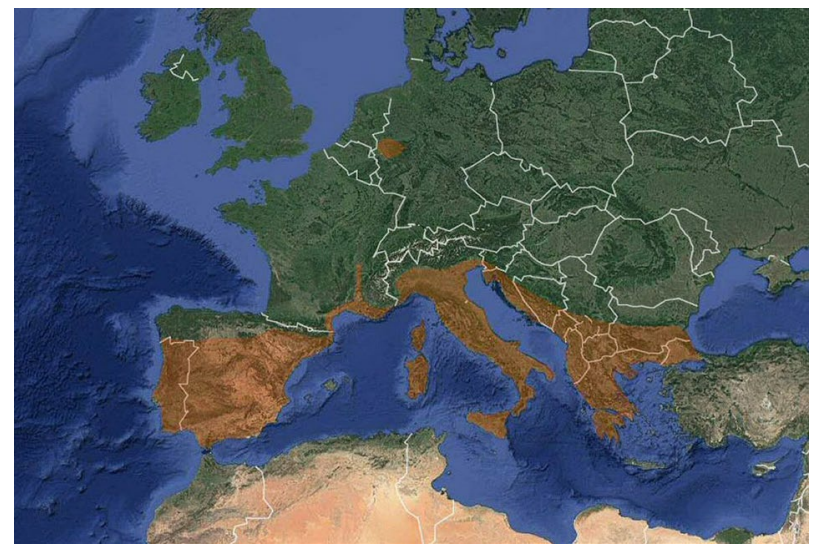

Figure 9. Répartition globale de Messor ibericus.

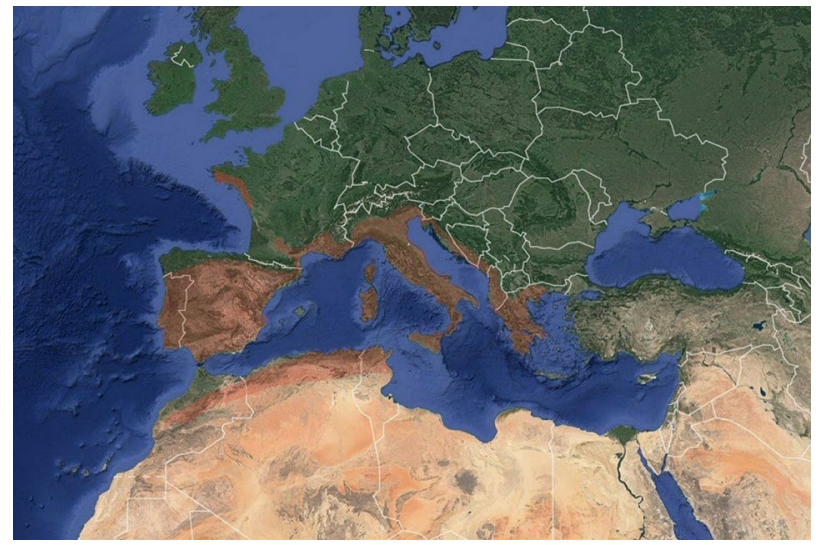

Figure 11. Répartition globale de Messor capitatus.

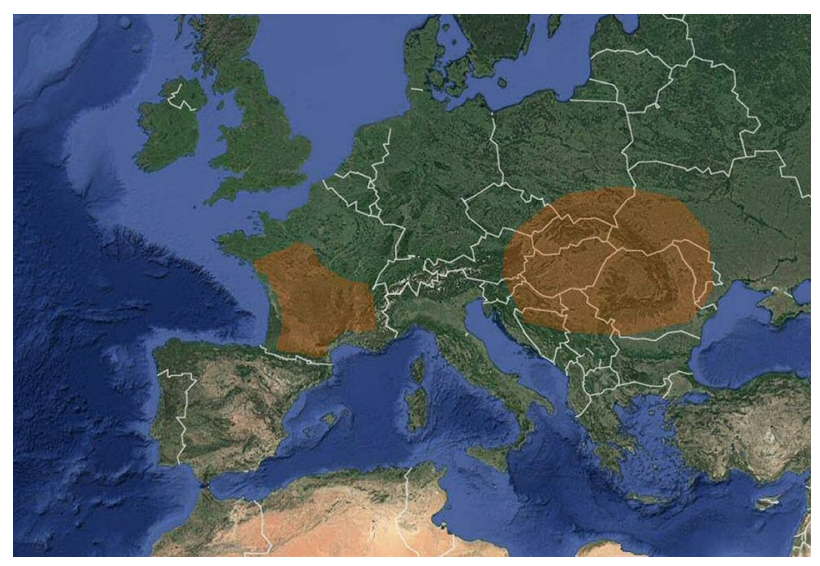

Figure 13. Répartition globale de Messor structor.

- Trois présentent une répartition strictement méditerranéenne : Messor barbarus (LINNÉ, 1767) (figures 5-6), Messor bouvieri BONDROIT, 1918 (figures 7-8) et Messor ibericus SANTSCHI, 1931 (figures 9-10).

- Une, Messor capitatus (LATREILle, 1798), est présente dans le Bassin méditerranéen, en Aquitaine et jusqu'en Bretagne (figures 11-12).

- La dernière espèce, Messor structor (LATREILLE, 1798) a une répartition plus continentale et scindée en deux (STEINER et al., 2018).

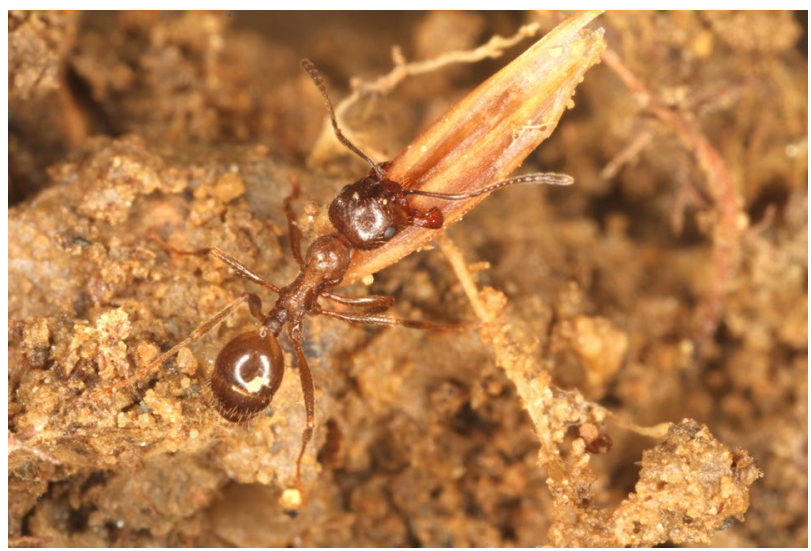

Figure 10. Ouvrière de Messor ibericus. Cliché C. LEBAS

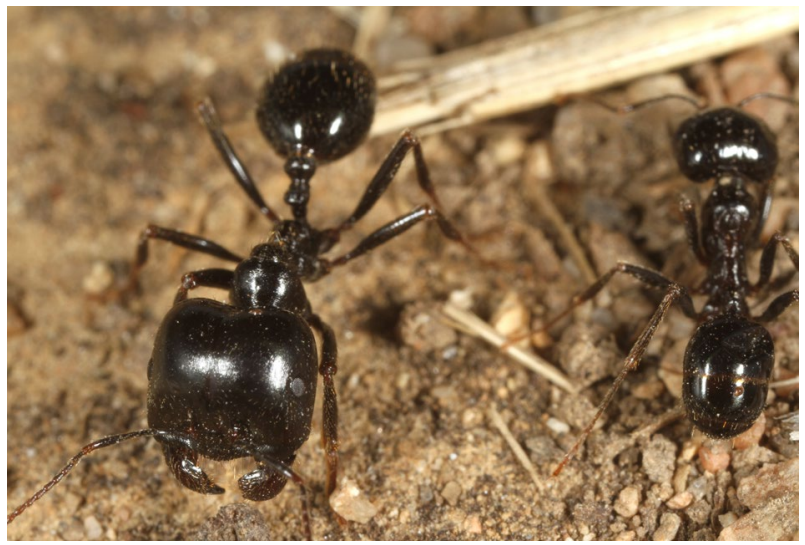

Figure 12. Ouvrière de Messor capitatus. Cliché C. LEBAS.

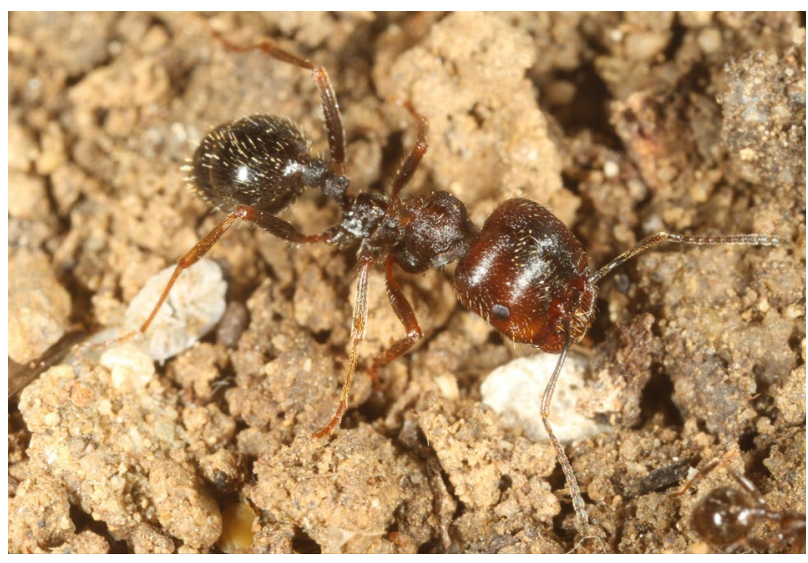

Figure 14. Ouvrière de Messor structor. Cliché C. LEBAS.

Il est à noter que le complexe d'espèces Messor structor $\mathrm{s}$. I. a été récemment éclairé et divisé en plusieurs espèces désormais reconnues (STEINER et al., 2018). En France, on trouve ainsi deux espèces, $M$. structor et $M$. ibericus, qui ont été longtemps confondues. La compréhension fine de leurs distributions respectives, avec à la clef la possibilité d'un éventuel chevauchement des aires de répartition, supposera, dans les années à venir, un effort poussé de prospection, d'échantillonnage et d'inventaire qu'il reste à fournir.

\section{MATÉRIEL ET MÉTHODES}

De juillet 2016 à octobre 2017, un recensement des Messor ici représentés par quatre espèces : $M$. barbarus, $M$. ibericus,
M. bouvieri et $M$. capitatus (COLLECTIF, 2021a) - a été réalisé dans les Pyrénées-Orientales. La recherche des chapelles, 
lieux susceptibles d'accueillir des populations de Messor, dans la plaine du Roussillon a été complété par la prospection des différents étages collinéens limitrophes. Le pointage de présence des Messor a été géoréférencé par GPS Garmin 20. Les ouvrières collectées ont été placées dans des tubes Eppendorf numérotés avec de l'alcool à $75^{\circ}$ et envoyées pour détermination à Christophe Galkowski (Antarea). La cartographie a été réalisée avec le logiciel QGIS (COLLECTIF, 2021b) et Google Maps pour les répartitions par espèces.

Cent vingt-sept sites historiques ont été visités (voir plus loin la figure 17): chapelles, ermitages, prieurés, sites antiques ou du Moyen-âge le plus souvent antérieurs au XII siècle. Les villes ou édifices plus récents voire contemporains n'ont pas été prospectés car la pression de l'homme y est trop forte pour que des fourmis granivores y subsistent.

\section{RÉSULTATS}

\section{Impact de l'altitude sur la distribution}

L'altitude maximale relevée pour chaque espèce de Messor dans les Pyrénées-Orientales est reportée dans la figure 15.

\section{Altitude maximale (m)}

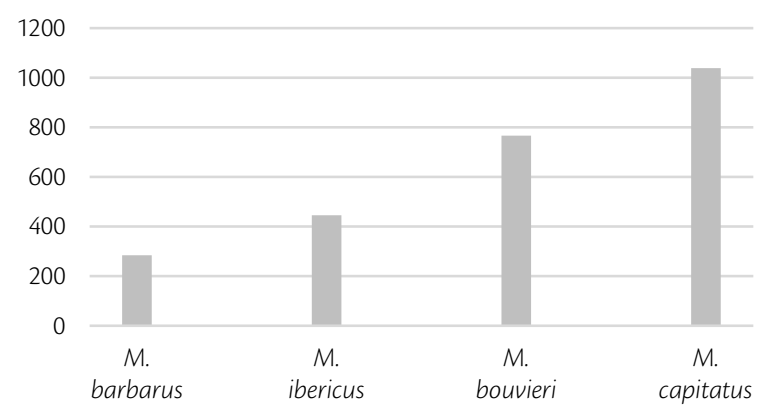

Figure 15. Altitude maximale de présence relevée lors de la présente étude pour les quatre espèces de Messor inventoriées dans les Pyrénées-Orientales.

Selon Cagniant \& ESPALAder (1998), Messor capitatus se rencontre au Maroc entre $1000 \mathrm{~m}$ et $1700 \mathrm{~m}$. En Algérie, elle nidifie de la côte à la montagne (COLLECTIF, 2018), essentiellement dans les grandes plaines céréalières (obs. pers.). Dans les Pyrénées-Orientales, on la trouve du littoral (Cap Béar) à 1038 m (Jujols) (N42 35.653 E2 21.294). Les stations situées aux altitudes les plus hautes se trouvent dans un environnement peu commun, à savoir des pelouses orientées plein sud avec une végétation maintenue rase par le pastoralisme.

La répartition de Messor barbarus est restreinte aux zones de plaine, n'atteignant pas $300 \mathrm{~m}$ pour les sites les plus élevés. Pourtant, l'altitude ne semble pas être un facteur limitant ou défavorable à son expansion puisque CAGNIANT \& ESPALADER (1998) la retrouvent jusqu'à 1300 m d'altitude au Maroc.

D'une manière générale, les pentes sont évitées pour tous les Messor. La difficulté réside probablement dans le port des graines qui seraient délicates à déplacer dans un tel contexte.

\section{Impact de la géologie sur la distribution}

Le bassin de l'actuel Roussillon (figure 16) a toujours fonctionné en régime amphibie. Les affleurements du plateau alternent entre un passé saumâtre et dulçaquicole (CLAUZON, 1987). Les fluctuations du niveau de la mer ont formé le cordon littoral par accumulation des sables et graviers.

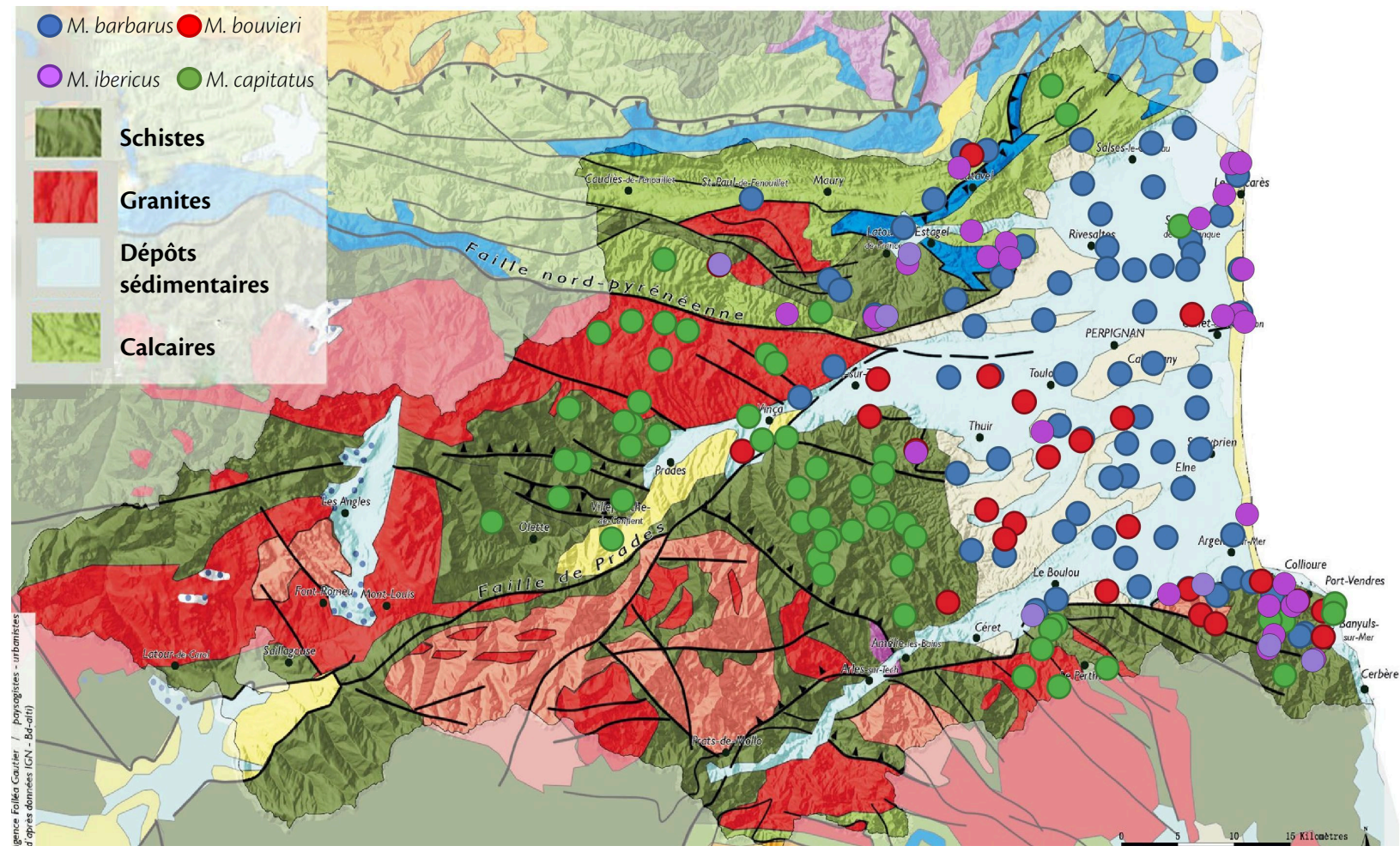

Figure 16. Géologie des Pyrénées-Orientales et répartition des fourmis du genre Messor. Données IGN BD ALTI. 
Ce mince lido fait une barrière aux eaux douces qui s'écoulent des reliefs puis s'accumulent ensuite sous forme d'étangs. Progressivement, le phénomène naturel de comblement de ces étangs par accumulations d'alluvions a effacé la physionomie du littoral lagunaire.

Messor barbarus et M. bouvieri ont ainsi pu s'installer sur le sol limoneux et caillouteux des dépôts sédimentaires de toute la plaine. Messor capitatus montre une préférence pour les schistes ainsi que les granites. Il est intéressant de noter que $M$. barbarus et $M$. capitatus se répartissent distinctement en fonction de la géologie (figure 16).

\section{Impact de la végétation sur la distribution}

Les Messor ne peuvent s'installer si la végétation est trop dense. La tenue et le transport des graines récoltées nécessite un terrain dégagé. Ainsi, la couverture forestière impacte davantage les fourmis que l'altitude (BLATRIX et al., 2016) et il est nécessaire que le milieu soit ouvert et ensoleillé. La chaleur est bénéfique au développement de la colonie et à la conservation des greniers constitués.

Quarante-quatre sites historiques, soit près de $34 \%$ de ceux visités, ne montrent pas de présence de Messor (figure 17). Cela peut être le fait d'un manque d'entretien (fauchage, sylviculture ou pastoralisme) conduisant à la fermeture naturelle du milieu. Les chênes verts essentiellement ont gagné l'espace offert initialement par les hommes avec leurs travaux agraires. Notons qu'une vallée, celle du Vallespir, semble trop fermée pour les Messor, avec de fortes pentes, une végétation très dense et un isolement sans voie d'accès avec la plaine du Roussillon.

Messor barbarus est localisé sur des terrains vagues, landes, vignes, talus, bords de route ou chemins. Cela correspond au milieu de la plaine et du littoral (figure 17). On peut la trouver en villages ou villes fortement urbanisés comme Perpignan intra-muros. L'espèce peut cohabiter sur le littoral, en arrière-dune, avec M. bouvieri.

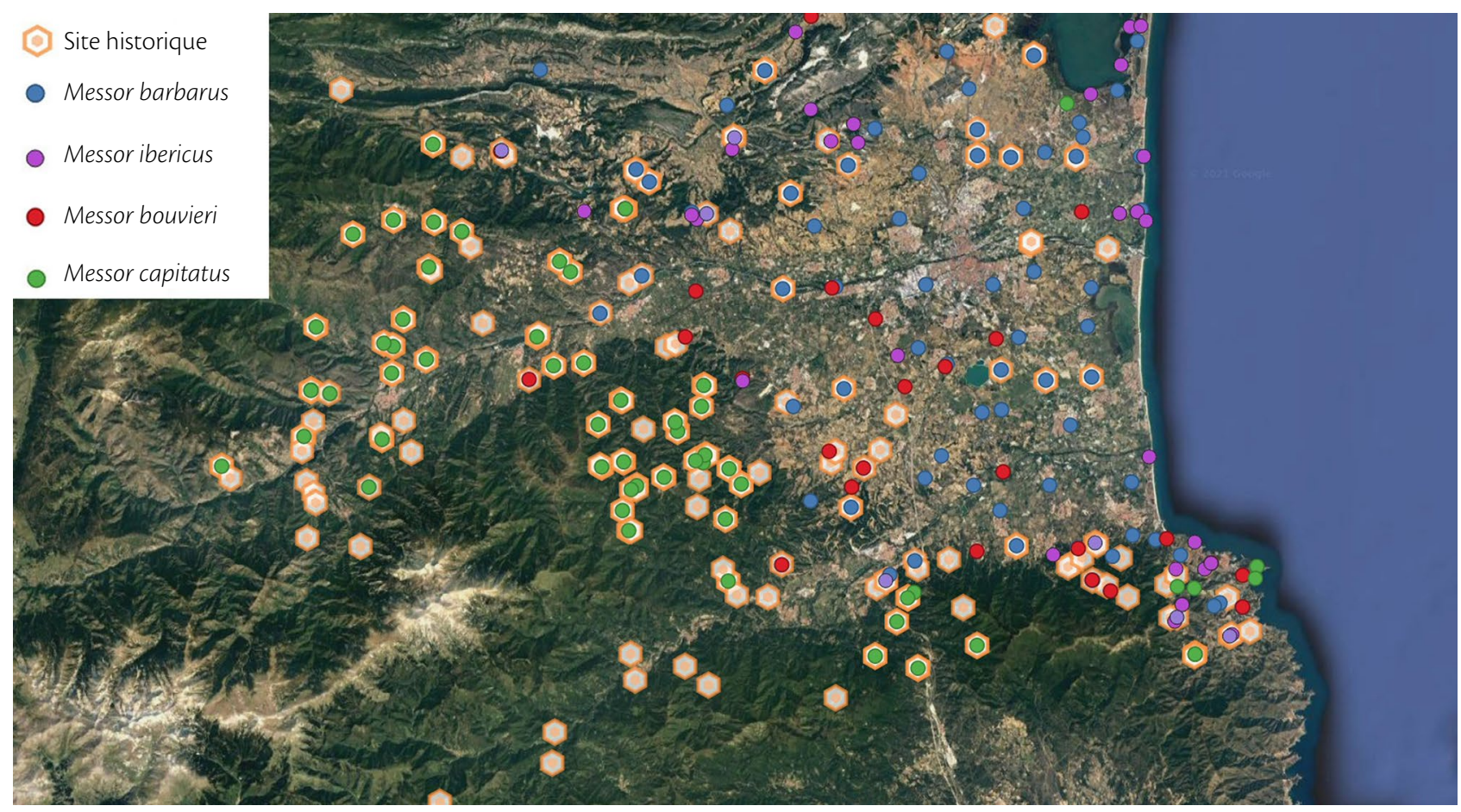

Figure 17. Répartition des fourmis du genre Messor dans les Pyrénées-Orientales en fonction des sites historiques visités.

Messor capitatus occupe systématiquement des sites anthropisés liées à une gestion des terres d'un passé ancien, abandonnés partiellement ou encore entretenus mais sans caractère expansif. Les parcelles demeurent réduites. Ce ne sont jamais des vignes. Pour l'essentiel, l'espèce occupe des placettes localisées au-delà des zones à chênes verts ou des suberaies se situant à la limite de l'étage euméditerranéen mais également en moyenne montagne (figures 17-18).

Messor bouvieri n'occupe que de petits secteurs de quelques dizaines de mètres carrés toujours ouverts et sans préférence particulière.

Messor ibericus nidifie dans les maquis à Ajoncs, les bords de chemin, talus, vignes et jardins (figures 17-18). Les plus grosses populations sont retrouvées au bord des agouilles.
Ces canaux, souvent à sec et bordés de cannes de Provence, permettent le ruissellement de l'eau vers la mer durant les très fortes pluies. Les berges sont sablonneuses et enrochées. En milieu xérique, les populations sont plus faibles.

La végétation et la différenciation entre Messor barbarus et M. capitatus est là aussi marquée (figure 18).

\section{Alimentation granivore}

Les céréales ont été domestiquées il y a plus de 9000 ans (CAMBECÈlES et al., 2012). Au Proche-Orient, en Syrie et Palestine, apparaissent des céréales sauvages (orge, engrain ou petit épeautre, blé amidonnier, etc.) et d'autres ressources végétales exploitables (lentille, pois, vesce et autres légumineuses) par l'homme. Ces plantes ont ensuite 


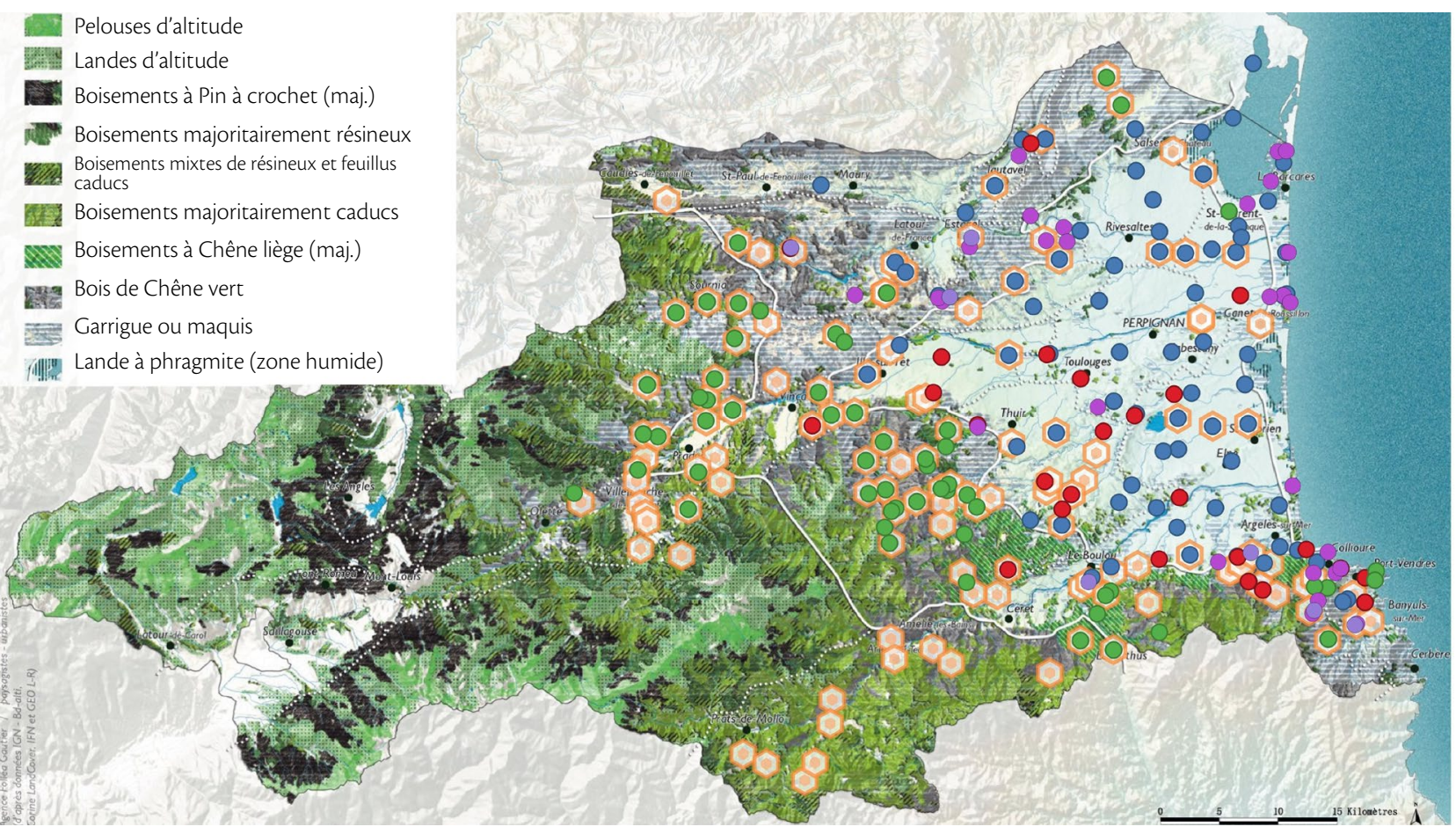

Figure 18. Répartition des fourmis du genre Messor dans les Pyrénées-Orientales en fonction de la végétation. Données Corine Land Cover, IFN et Géo LR. Les points sont les mêmes que sur la figure 17.

été déplacées par l'homme jusqu'en Europe occidentale, souvent accompagnées par des plantes messicoles ou adventices durant le bronze ancien et la civilisation minoenne il y a 6000 ans (CAMBECÈLES et al., 2012) (figure 19).

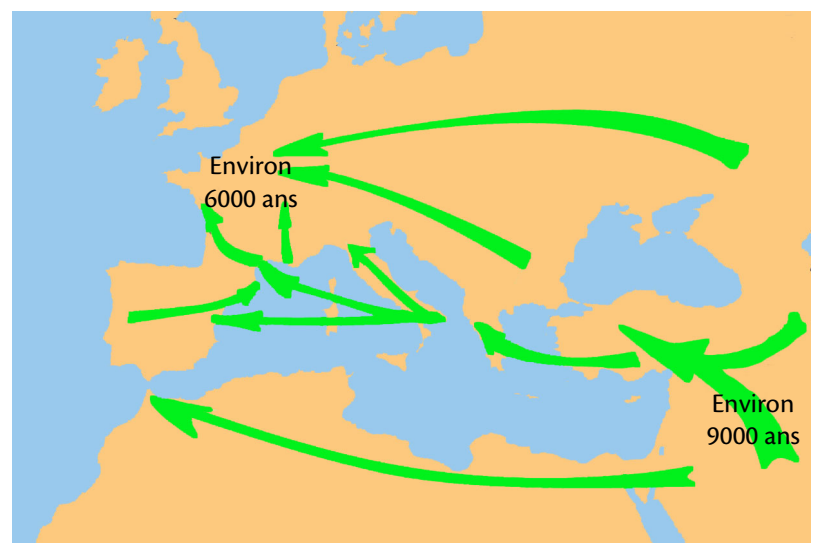

Figure 19. Flux anthropiques historiques de dispersion des plantes messicoles.

Synthétisé d'après CAMBECÈLES et al. (2012).

Ce sont les graines de toutes ces plantes qui sont les ressources nutritives des fourmis Messor. Le nom de ce genre de fourmis communément appelées « fourmis moissonneuses » vient du latin messis qui signifie moisson. Les fourmis Messor sont des moissonneuses opportunistes, les graines les plus récoltées étant celles qui sont les plus nombreuses dans leur milieu (CERLAN \& DEYLE, 1990).

Le genre Messor est associé à une alimentation stricte. Contrairement à de nombreux genres chez les fourmis, il n'y a pas de jabot social et l'absence de trophallaxie est compensé par une prédigestion de la nourriture. Le système de filtration des substances est différent de celui des fourmis polyphages. Les fourmis ne peuvent absorber que du liquide. Le genre Messor possède un mécanisme d'apport de salive sur les graines pré-mâchées qui provoque une hydrolyse de l'amidon. Celui-ci est ensuite absorbé sous forme de sucre après une digestion extra-orale (DELAGE, 1962).

Les Messor sont considérés comme apparentés aux Aphaenogaster (BERNARD, 1985 ; CAGNIANT, 2006 ; PLOWES et al., 2015) et M. structor serait l'espèce la moins spécialisée des Messor à laquelle on pourrait certainement ajouter l'espèce très proche M. ibericus (STEINER et al., 2018). Leur ubiquité irait de pair avec leur faible degré de spécialisation (DELAGE, 1968). On peut ainsi trouver dans leurs nids des graines issues d'autres plantes que des messicoles, comme celles de la garrigue (ciste, genêt, myrte, bruyère arborescente...) (figure 20).

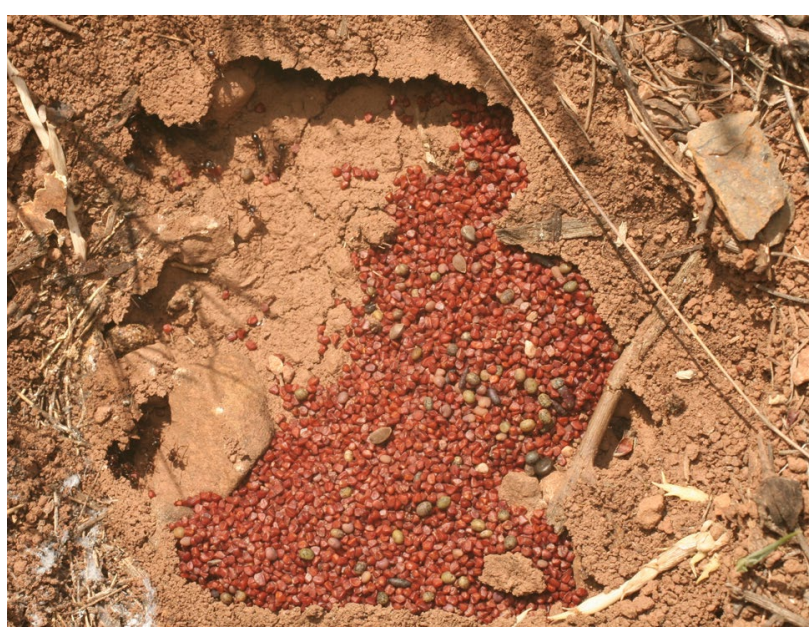

Figure 20. Grenier à grain de Messor ibericus. Cliché C. LEBAS.

Les populations de Messor barbarus sont très importantes sur les sites où elle est installée. Ainsi, dans d'autres travaux, il a été comptabilisé en moyenne 468 nids par hectare (BARALBAR et al., 2011). Les processions d'ouvrières, récoltant 
et fourrageant collectivement les semences, forment des pistes stables et permanentes pouvant perdurer pendant l'hiver. Les pistes peuvent atteindre $39 \mathrm{~m}$ pour une largeur de 10 à $20 \mathrm{~cm}$; ces paramètres sont modulés par la nature et la disponibilité des ressources ainsi que le nombre d'entrées du nid (LOPEZ et al., 1994). La densité des nids peut être importante, avec beaucoup de proximité, sans pour autant entraîner de phénomènes de compétition. Selon DETRAIN et al. (1996), M. barbarus est généraliste et ne présente pas d epréférence alimentaire marquée.

La fourmi Messor capitatus, quant à elle, procède la plupart du temps à une récole des graines par recherche individuelle sans effectuer de longues colonnes (ARMAN et al., 2010).
Cette fourmi est inféodée au Festucetum que l'on retrouve comme plante fourragère dans les pâtures (DARCHEN, 1976). Si Messor capitatus loge sur des sites anthropisés, on peut l'expliquer par son alimentation sélective de ces denrées liées à l'activité pastorale. Il suffit que la physionomie d'un site soit modifiée pour que les ressources s'amenuisent, mettant alors en péril la présence de l'espèce à cet endroit. Dix-sept sites historiques occupés par M. capitatus sont des pelouses à Festucetum où pâturent des ruminants.

Finalement, Messor barbarus est une espèce compétitive, opportuniste et tolérante. Par contraste, $M$. capitatus est plus fragile et inféodée à l'homme dans sa gestion agricole des lieux.

\section{DISCUSSION DES MODALITÉS DE DISPERSION DES MESSOR AU COURS DES ÂGES}

\section{Liens entre anthropisation et distribution des Messor dans les Pyrénées-Orientales}

À l'instar des fourmis, les hommes montrent des préférences dans le choix et l'exploitation des terres cultivables, privilégiant l'occupation de sites offrant un espace ouvert bien ensoleillé dans un cadre de verdure. Les pelouses d'extension modérée sont souvent un lieu d'implantation de chapelles et autres édifices religieux ou non. II peut alors y avoir convergence d'intérêts entre les fourmis et l'homme sans relations compétitives apparentes. Au contraire, une forme proche du commensalisme semble s'établir au profit des Messor. En 1985, BERNARD avançait néanmoins que le tiers des récoltes des hauts plateaux d'Algérie pouvait passer dans les greniers des fourmis, soit une perte tout de même substantielle de denrées alimentaires pour l'homme. D'un autre côté, les fourmis Messor, dans leur recherche de graines, favorisent la dissémination des semences qu'elles ont triées (AZCÁRATE et al., 2005).

Messor capitatus semble attester de cette convergence par sa capacité à exploiter les sites anthropisés historiques: $91,8 \%$ des sites montrant des traces d'activités humaines modérées et traditionnelles, passées ou actuelles, sont occupés par cette espèce ( 56 occurrences sur 61 inventaires). Messor barbarus, en revanche, n'exploite ce type de sites que dans $19,7 \%$ des cas (19 pointages pour 77 inventoriés). Sa répartition se limite à la plaine alors que $M$. capitatus en est exclue. Dans les milieux à urbanisation forte et récente, M. barbarus occupe la place sans partage.

De ces deux espèces granivores, l'une, $M$. barbarus tolère à la fois l'urbanisation, les pratiques viticoles, les terres non exploitées ou abandonnées ainsi que les conditions du proche littoral. L'autre, $M$. capitatus, est inféodée à des pratiques agraires et de pastoralisme à petite échelle. Son maintien sur une station donnée devient menacé dans le cas d'un abandon ou d'une modification des pratiques agropastorales locales (DARCHEN, 1976).

\section{Contribution à la compréhension du processus historique de dispersion des Messor}

Des études récentes permettent d'éclairer la phylogéo- graphie et la dynamique de conquête des territoires par les Messor, qu'il s'agisse d'études génétiques comme celle de STEINER et al. (2018), ou d'une approche biogéographique plus classique basée sur une synthèse de données bibliographiques, comme l'ont fait TINAUT \& RUANO (2021). Ces travaux suggèrent des routes de dispersion de la myrmécofaune qui vont dans le même sens que les observations présentées dans cette étude.

Comme nous l'avons vu en introduction, les Messor présentes en France possèdent une limite de répartition allant de l'Anatolie aux contours du Bassin méditerranéen.

Un clivage proposé par CAGNIANT (1973) sépare les espèces en deux ensembles par un axe qui va de la basse vallée du Rhône à la plaine du Pô. À l'est de cet axe, on ne trouve que Messor wasmanni (figure 1) et M. minor (figure 3). Messor bouvieri (figure 7) et $M$. barbarus (figure 5) ne sont rencontrés qu'à l'ouest. L'explication réside peut-être dans l'isolement de la Péninsule italique à la fin du Miocène, lorsque les vallées du Pô et du Rhône ont été submergées par la mer (TABERLET, 1998 ; TABERLET et al. 1998).

Messor structor (figure 13) présente une distribution davantage continentale, scindée en deux de part et d'autre des Alpes par la séparation sus-citée. La vicariance nette et remarquable caractérisant cette aire de répartition suggère une colonisation des espaces par différentes voies, l'Europe centrale ayant pu bénéficier d'un refuge lors du Pléistocène (STEINER et al., 2018). Le contournement des Alpes à l'Ouest par les ancêtres des populations allemandes semble correspondre à des voies de colonisation déjà décrites pour d'autres espèces, comme le papillon Polyguatus coridon (SCHMITT et al., 2002) ou certaines libellules (STENBERG, 1998). Pour SCHLICK-STEINER (2006), les ancêtres de la population autrichienne et tchèque ont progressé par un pontage par l'est (figure 20). Notons que toutes ces voies de colonisation sont présentes dans la synthèse de TABERLET (1998).

Deux espèces font exception à ce clivage : Messor ibericus et M. capitatus.

La répartition est (quasiment) strictement méditerranéenne pour Messor ibericus (figure 9). Elle est établie sur le pourtour 


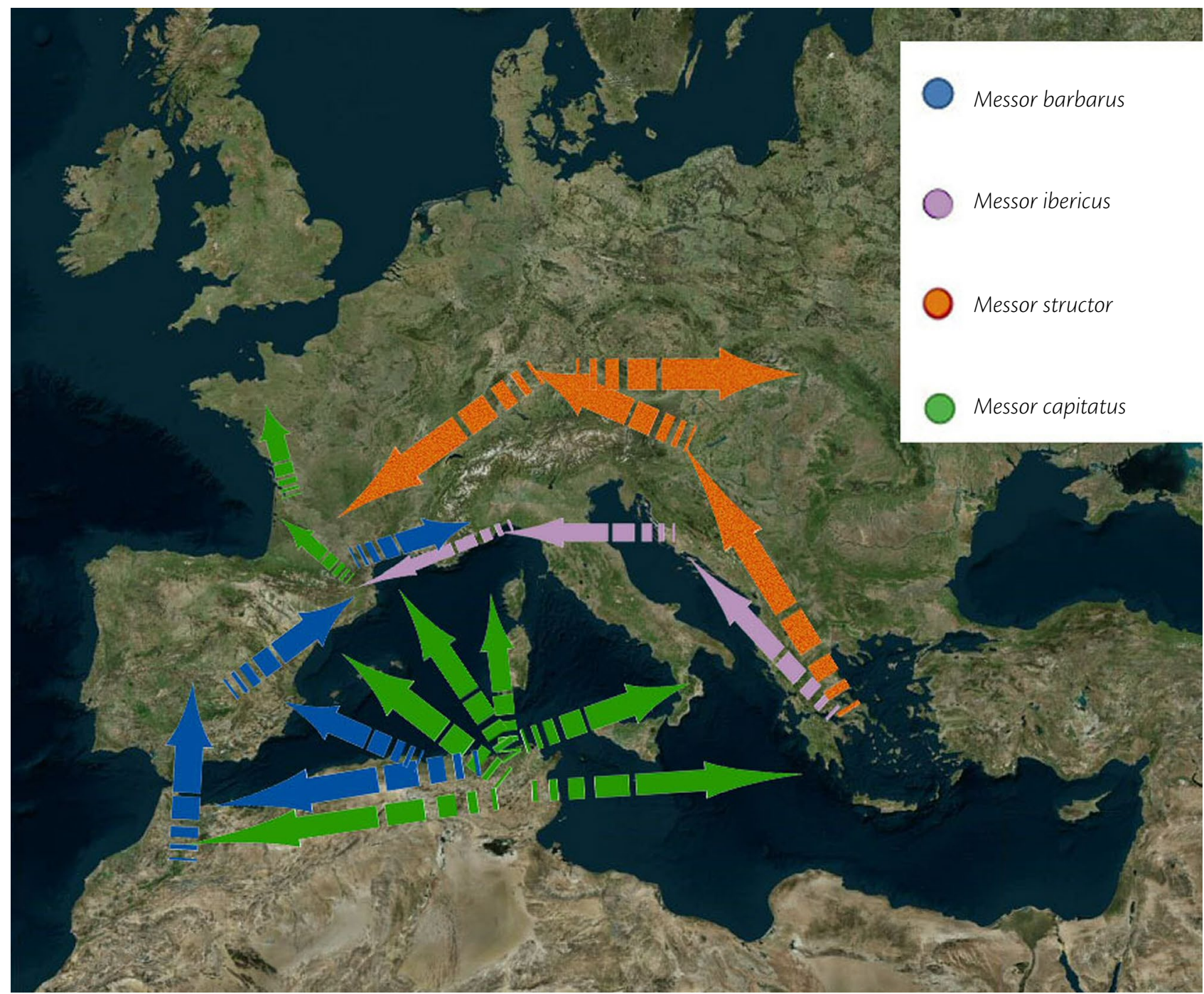

Figure 21. Routes hypothétiques de colonisation de l'aire de répartition globale des espèces de Messor représentées dans les Pyrénées-Orientales.

nord du Bassin méditerranéen et ne semble pas avoir été affectée par un clivage. On la trouve toutefois dans une poche en Allemagne au sein de la province romaine de Germanie inférieure (STEINER et al., 2018). La présence romaine durant l'Antiquité permet de penser que l'espèce a probablement suivi les voies de transport des céréales pour $s^{\prime} y$ installer (figure 21). Son régime alimentaire varié lui permet de s'adapter à tous les milieux. Comme Messor structor, elle n'est donc pas inféodée strictement aux cultures des hommes.

Si le transport des fourmis par les activités humaines a éventuellement pu s'effectuer au cours des âges, l'absence de ressources nutritives pour des granivores autres que Messor structor et $M$. ibericus a probablement limité drastiquement leur développement territorial. II était du reste sans doute difficile pour des fourmis non natives d'arriver avant ces mouvements.

Messor capitatus semble rare à l'ouest du Maghreb où on la retrouve seulement en montagne (CAGNIANT \& ESPADALER, 1998). En revanche, sa présence est plus importante en Algérie et en Tunisie, de la côte à la moyenne montagne (CAGNIANT \& ESPADALER, 1998). Ces mêmes auteurs la considèrent moins commune qu'en Europe méridionale. Elle n'a probablement pas rencontré les mêmes facteurs environnementaux favorables à son développement, notamment par son alimentation plus stricte. La colonisation de l'Europe de cette espèce s'est probablement opérée par voie maritime via la Sicile, la Sardaigne et la Corse, au moyen de bateaux en partance de la zone tunisienne et de son plus grand port commerçant, Carthage (figure 20). Les premiers hommes à être arrivés par la mer dans le Roussillon sont les Grecs au Ve siècle avant J.-C. (UGOLINI, 2010). La distribution de l'espèce en Méditerranée semble suivre le parcours des céréales et celui du commerce de Mycéniens (BALMUTH et al. 1998). Au-delà du Bassin méditerranéen, elle a partiellement colonisé le Bassin aquitain et la côte bretonne. DELAGE (1968) suggère une similitude entre distribution de $M$. capitatus et les grands cortèges floristiques du Bassin aquitain. Selon elle, les plantes méditerranéennes prévalent sur les euro-asiatiques, sudeuropéennes ou autres dans le régime alimentaire de l'espèce. Si on observe le flux historique des plantes messicoles (figure 18), il semble bien ressembler à la dispersion des $M$. capitatus au niveau du Bassin méditerranéen (figure 21). Dans le Roussillon, tous les sites historiques où Messor capitatus est présent sont antérieurs au XII siècle. Ceci correspond à la période d'occupation du Roussillon par les Wisigoths puis les Francs à l'époque carolingienne (DURLIAT, 1969) En se sédentarisant, les hommes ont ouvert les milieux par leurs pratiques agraires. 
Des religieux, souvent ermites, ont évangélisé ces sites isolés (SERRES-BRIA, 2004). L'association entre les sites religieux et la présence de Messor capitatus est donc probablement à expliquer par cette dynamique humaine, ces fourmis échappant ainsi à l'insalubrité de la plaine à l'époque (CLAUZON, 1987 ; MARTINEZ \& ROSSIGNOL, 1975).

Messor barbarus s'est probablement installé dans la Plaine du Roussillon après son assèchement à partir du XIle siècle (CAUCANAS, 1995). L'espèce serait originaire de l'est de la Berbérie, plus précisément du Constantinois (CAGNIANT, 1962), là où justement $M$. capitatus est plus rare. Messor barbarus aurait ensuite sans doute progressé par l'Atlas pour remonter ensuite par l'Espagne (figure 20), ce que CAGNIANT \& ESPADALER (1998) définissent comme étant une espèce maghrébine à extension européenne.

Messor bouvieri (figure 7) serait une endémique ibérique qui a glissé sur le littoral méditerranéen français. Comme pour Messor structor et $M$. ibericus, son large régime alimentaire peu exigeant ne dépendant pas des activités humaines
(FERNÁNDEZ-ESCUDERO \& TINAUT, 1993) a vraisemblablement facilité son expansion.

\section{Perspectives de conservation de Messor capitatus}

Messor capitatus est très dépendant des activités humaines comme la culture des plantes messicoles et fourragères ainsi que de l'élevage tant qu'ils restent traditionnels. Si la reforestation, l'urbanisation, la culture de la vigne ou de vergers gagnent du terrain, l'espèce perd les conditions de son maintien. La conservation de ces sites et de leurs pratiques agraires sont donc des éléments majeurs à considérer dans une optique de conservation de l'espèce.

En Corse, mes observations personnelles m'amènent à penser que la même étude pourrait être effectuée. $M$. capitatus y nidifie sur les vestiges romains, les édifices religieux ou les sites de transhumance ancestraux. Elle y entre en revanche en conflit avec $M$. wasmanni (qui est très proche de M. barbarus par sa biologie).

\section{REMERCIEMENTS}

Que Christophe GALKOWSKI soit remercié pour tous les échantillons que j'ai pu lui adresser à identifier. Mes remerciements s'adressent également à Philipe BOURLET pour ses recommandations à la relecture du document et à Laurent CoLINDRE pour ses encouragements. Enfin, j'exprime ma reconnaissance à Tanguy JEAN et Benoît GESLIN pour leurs précieux conseils et leur accompagnement patient et pédagogique. 


\section{RÉFÉRENCES}

ArmAn, X., J. RetANA, A. RoDrIGo \& X. CerdÁ (2010). Foraging behaviour of harvesting ants determines seed removal and dispersa. Insectes Sociaux, 57(4): 421-430. https://doi.org/10.1007/s00040-0100100-7

AzacÁrate, M., L. Arqueros, A. M. Sánchez \& B. Peco (2005). Seed and fruit selection by harvester ants, Messor barbarus, in Mediterranean grassland and scrubland. Functional Ecology, 19(2): 273-283. https://doi.org/10.1111/j.0269-8463.2005.00956.x

BALMUTH, M. S. \& R. H. TYKOT. (ed.) (1998). Sardinian and Aegean Chronology. Oxbow, Oxford, $416 \mathrm{pp}$.

Baraibara, B., J. TORRA \& P. R. WestermAn (2011). Harvester ant (Messor barbarus (L.)) density as related to soil properties, topography and management in semi-arid cereals. Applied Soil Ecology, 51: 60-65. https://doi.org/10.1016/j.apsoil.2011.08.012

BERNARD, F. (1985). Recherches sur l'évolution des fourmis moissonneuses (Hym. Formicidae). Actes des Colloques Insectes sociaux, 2: 45-55.

Blatrix, R., C. Lebas, C. Galkowski, P. Wegnez, R. Pimenta \& D. MORICHON (2016). Vegetation cover and elevation drive diversity and composition of ant communities (Hymenoptera: Formicidae) in a Mediterranean ecosystem. Myrmecological News, 22: $119-127$.

https://myrmecologicalnews.org/cms/index.php?option=com_c ontent\&view=category\&id=633\&ltemid=366 [accessed 15 April 2021]

CAGNIANT, H. (1964 ["1962"]). Étude de quelques fourmis marocaines. Statistique provisoire des Formicidae du Maroc. Bulletin de la Société d'Histoire Naturelle de l'Afrique du Nord, 53: 83-118. https://doi.org/10.5281/zenodo.26555

CAGNIANT, H. (1973). Le peuplement de fourmis des forêts algériennes. Écologie, biocénotique, essai biologique. Thèse de doctorat, Université Paul Sabatier, Toulouse (France), 463 pp.

CAGniant, H. (2006). Messor boyeri n. sp. du Maroc. Orsis, 21: 7-13. https://antcat.org/references/142544 [accessed 15 April 2021]

CAGNIANT, H. \& X. Espadaler (1998 ["1997"]). Le genre Messor au Maroc (Hymenoptera : Formicidae). Annales de la Société Entomologique de France (N.S.), 33: 419-434.

https://antcat.org/references/130880 [accessed 15 April 2021]

CAMBECĖDES, J., G. LARGIER \& A. LOMBARD (2012). Plan national d'actions en faveur des plantes messicoles. Conservatoire botanique national des Pyrénées et de Midi-Pyrénées - Fédération des Conservatoires botaniques nationaux - Ministère de l'Écologie, du Développement durable et de l'Énergie, Paris, 242 pp. https://www.ecologie.gouv.fr/sites/default/files/PNA_Plantesmessicoles_2012-2017.pdf [accessed 15 April 2021]

Cerdan, P. \& G. Deleye (1990). La consommation des graines par les fourmis Messor barbarus et Messor sanctus dans la plaine de Crau, Actes des Colloques Insectes Sociaux, 6: 165-130.

ColleCTIF, 2018. Antmaps. https://antmaps.org/ [accessed 15 June 2018]

ColleCTIF, 2021a. Répartition des espèces françaises de Fourmis. Association AntArea. Canohès (France).

https://antarea.fr/fourmi/?repartition/repertition-especes.html [accessed 15 April 2021]

COLLECTIF, 2021b. QGIS. Système d'information géographique libre et open source. Travail collaboratif mondial. https://www.qgis.org/fr/site/ [accessed 15 April 2021]

CLAUZON, G. (1987). Le détritisme néogène du bassin du Roussillon (Pyrénées-Orientales, France). Géologie Alpine, special issue 13: 427-441. https://hal-insu.archives-ouvertes.fr/insu-00512531/ [accessed 15 April 2021]

DARCHEN, B. (1976). Disparition d'un biotope à Messor capitatus LATR. (Hyménoptères, Formicidés) consécutive à l'évolution naturelle d'un causse en Périgord noir. Bulletin d'Écologie, 7(2): 215-220. https://www.dictionnaire-amoureux-desfourmis.fr/Noms\%20propres/D/Darchen\%20Bernadette/B\%20D archen\%20Messor\%20capitatus.pdf [accessed 15 April 2021]
DeLAGE, B. (1962). Recherches sur l'alimentation des fourmis granivores Messor capitatus LATR. Insectes sociaux, 9(2): 137-143. https://doi.org/10.1007/BF02224260

DELAGE, B. (1968). Recherches sur les fourmis moissonneuses du Bassin aquitain : écologie et biologie. Bulletin Biologique de la France et de la Belgique, 102: 315-367.

https://www.dictionnaire-amoureux-desfourmis.fr/Noms\%20propres/D/Darchen\%20Bernadette/B\%20D elage\%20messor\%20capitatus.pdf [accessed 15 April 2021]

Detrain, C., M. Versaen \& J. M. Pasteels (1996). Récoltes de graines et dynamique du réseau de pistes chez la Fourmi moissonneuse Messor barbarus. Actes des Colloques Insectes sociaux, 10: 157160.

Durlat, M. (1963) Histoire du Roussillon. Presses universitaires de France, Paris, $126 \mathrm{pp}$.

FERNÁNDEZ-ESCUDERO I. \& A.TINAUT (1993). Alimentación no granívora en Messor bouvieri BONDROIT, 1918 y Messor barbarus (L. 1767) (Hymenoptera:Formicidae). Boletín de la Asociaión española de Entomología, 17(2): 247-254.

https://docplayer.es/145829648-Alimentacion-no-granivora-enmessor-bouvieri-bondroit-1918-y-messor-barbarus-1-1767hymenoptera-formicidae.html [accessed 15 May 2021]

Guénard, B., M. Weiser, K. Gomez, N. Narula, E. P. EConomo (2017) The Global Ant Biodiversity Informatics (GABI) database: a synthesis of ant species geographic distributions. Myrmecological News, 24: 83-89. https://doi.org/10.25849/myrmecol.news_024:083

Lebas, C., C. Galkowski, R. Blatrix \& P. Wegnez (2016). Fourmis d'Europe Occidentale. Delachaux et Nestlé, Paris, 415 pp.

LEBAS, C. (2021). Messor capitatus et la religion dans les Pyrénées Orientales. Blogspot, Blogger - Google. https://messor-capitatushistoire.blogspot.com/ [accessed 15 April 2021]

Lopez, F., F. J. Acosta \& J. M. SerRano (1994). Guerilla vs. phalanx strategies of resource capture: growth and structural plasticity in the trunk trail system of the harvester ant Messor barbarus. Journal of Animal Ecology, 63(1): 127-138. https://doi.org/10.2307/5589

PLOWES, N., R. A. JOHSON \& B. HÖLLDOBLER (2013). Foraging behavior in the ant genus Messor. Myrmecological News, 18: 33-49. https://myrmecologicalnews.org/cms/index.php?option=com_c ontent\&view=category\&id=560\&ltemid $=362$ [accessed 15 April 2021]

SChmitt, T., A. Gießl. \& A. SeITZ (2002). Postglacial colonisation of western Central Europe by Polyommatus coridon (PODA 1761) (Lepidoptera: Lycaenidae): evidence from population genetics. Heredity, 88: 26-34. https://doi.org/10.1038/sj/hdy/6800003

Schlick-Steiner, B. C., F. M. Steiner, H. Konrad, B. Markó, S. Csösz, G. Heller, B. Ferencz, B. Sipos, E. Christian \& C. Stauffer (2006). More than one species of Messor harvester ants (Hymenoptera: Formicidae) in Central Europe. European Journal of Entomology, 103(2): 469-476. https://doi.org/10.14411/eje.2006.060

Steiner, F. M., S. Csösz, B. Markó, A. Gamischad, L. Rinnhofera, C. Folterbauera, S. Hammerlea, C. Stauffere, W. Arthofera \& B. C. SCHLICK-STEINER (2018). Turning one into five: Integrative taxonomy uncovers complex evolution of cryptic species in the harvester ant Messor "structor". Molecular Phylogenetics and Evolution, 127: 387-404

https://doi.org/10.1016/j.ympev.2018.04.005

SeRres-BRIA, R. (2003). Les Ermitages du Roussillon et leurs ermites. Société agricole, scientifique et littéraire des Pyrénées-Orientales, Perpignan, $191 \mathrm{pp}$.

TABERLET, P. (1998). Biodiversity at the intraspecific level: The comparative phylogeographic approach. Journal of Biotechnology, 64(1): 91-100. https://doi.org/10.1016/S0168-1656(98)00106-0

TABerlet, P., L. Fumacalli, A.-G. Wust-SAuCy \& J.-F. Cosson (1998). Comparative phylogeography and postglacial colonization routes in Europe. Molecular Ecology, 7(4): 453-464. https://doi.org/10.1046/j.1365-294x.1998.00289.x 
TINAUT, A. \& F. RuANO (2021). Biogeography of Iberian Ants (Hymenoptera: Formicidae). Diversity, 13(2): 88 [25 pp.]. https://doi.org/10.3390/d13020088
UGolıNI, D. (2010). Présences étrangères méditerranéenne sur la côte du Languedoc-Roussillon durant l'âge du Fer : de la fréquentation commerciale aux implantations durables. Pallas, Revue d'Études Antiques, 84: 83-110. https://doi.org/10.4000/pallas.3369

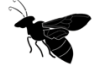

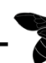


OSMIA est éditée par I'Observatoire des Abeilles (OA), une association loi 1901 d'apidologues (ou mellitologues) d'Europe francophone qui œeuvrent pour la connaissance et la protection des Abeilles sauvages

Les articles sont:

- publiés uniquement en ligne

- disponibles en open access,

- indexés / archivés par Crossref Zoobank HAL, Zenodo OpenAIRE, Google Scholar et Web of Science (Clarivate) OpenAIRE, Google
[Zoological Record]

- respectueux des recommandations de la Commission internationale de Nomenclature zoologique (ICZN),

sous Licence Creative Commons Attribution International CC BY 4.0 qui autorise la reproduction et la diffusion du document, à condition d'en citer explicitement la source,

- librement déposables sur des sites internet ou des plateformes d'archivage.

(!) Les documents d'autres sources et non distribuées sou licence libre sont reproduits après autorisation (à demander par les auteurs) et demeurent la propriété des auteurs ou éditeurs originaux.

(!) Le contenu publié est sous l'entière responsabilité des (!) Le conte
auteurs.

OSMIA est conçue pour une impression recto-verso en haute résolution. Les bibliothèques publiques, les laboratoires, les muséums et les associations sont invités à imprimer et conserver une version papier de la revue.
OSMIA is published by the Observatory of Bees (OA), a nonprofit society of apidologists (or mellitologists) from Frenchspeaking Europe who work together for the knowledge and protection of wild bees.

The items are:

- published only online

- available in open access

- indexed / archived by Crossref, Zoobank, HAL, Zenodo, OpenAIRE, Google Scholar and Web of Science (Clarivate) OpenAIRE, Google Sc

- respectful of the recommendations of the International Commission for Zoological Nomenclature (ICZN)

under Creative Commons Attribution Licence International CC BY 4.0 which authorises the reproduction and distribution of the document, provided the source is explicitly cited

- freely depositable on personal or institutional websites and archiving platforms.

(!) Documents from other sources and not distributed under a free license are reproduced after authorisation (to be requested by the authors) and remain the property of the original authors or publishers.

(!) The published content is the sole responsibility of the (!) The

OSMIA is designed for high-resolution printing on both sides. Public libraries, laboratories, museums, and societies are invited to print and keep a paper version of the journal.
Directeur de la publication - Editor-in-chief Benoit GESLIN

Comité éditorial • Editorial Board

Matthieu AUBERT • Floriane FLACHER • Mehdi ISSERTES

Tanguy JEAN • Léa LEMAIRE

Mise en page $\cdot$ Layout

Mehdi ISSERTES • Tanguy JEAN • Léa LEMAIRE

Comité de lecture • Scientific committee 2021 https://www.osmia-journal-hymenoptera.com/equipe-team.html

Soumission d'articles • Submission of items osmia.editor@gmail.com

Recommandations aux auteurs . Recommendations to authors https://www.osmia-journal-hymenoptera.com/auteurs-authorsinstructions.html

Observatoire des Abeilles

68 rue du Onze Novembre

F - 59148 Flines-lez-Râches (France)

https://oabeilles.net/

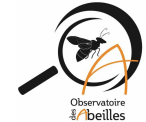

OPEN

Access 